\title{
Modelagem da Propagação da Doença de Chagas por triatomíneos
}

\author{
Vanessa Steindorf* \\ Centro de Matemática, Computação e Cognição, UFABC \\ 09210-580, Santo André, SP \\ E-mail: vanessasteindorf@hotmail.com \\ Norberto A. Maidana \\ Centro de Matemática, Computação e Cognição, UFABC \\ 09210-580, Santo André, SP \\ E-mail: norbertomaidana@gmail.com
}

\section{RESUMO}

Este trabalho tem como objetivo estudar a propagação da doença de Chagas, através de um modelo matemático. Deste modo, para estudar a dinâmica que descreve a propagação desta doença, na população humana, por vetores transmissores (os triatomíneos), propomos um modelo de equações diferenciais ordinárias, levando em consideração a transmissão direta da doença, assim como a transmissão vertical, com o objetivo final de propor estratégias de controle do vetor transmissor em uma comunidade.

A doença de Chagas é reconhecida como um dos maiores problemas de saúde em países da América Latina. É transmitida por insetos hematófagos que pertencem à família Reduviidae, subfamília Triatominae, conhecidos no Brasil como barbeiro [1]. De acordo com relatório da OMS de 2007, nos países endêmicos havia aproximadamente 8 milhões de pessoas infectadas. Portanto, é indispensável que esta doença não seja negligenciada e que, preocupações como manter o controle da doença são necessárias para evitar possíveis situações epidemiológicas.

A doença de Chagas é causada pelo protozoário flagelado Trypanosoma Cruzi, da família Trypanosomatidae. No ser humano, a transmissão do Trypanosoma Cruzi, ocorre principalmente por meio de um vetor, os triatomíneos.

As principais formas de transmissão da doença de Chagas para o homem são:

i) Transmissão Vetorial

Acontece pelo contato do homem com as excretas contaminadas com Trypanosoma Cruzi dos triatomíneos. A infecção ocorre quando o barbeiro, ao picar e sugar o sangue do homem, defeca, eliminando formas infectantes de tripomastigotas que penetram pelo orifício da picada.

ii) Transmissão Transplacentária

A principal via da transmissão vertical é a transplacentária ou congênita e ela pode ocorrer em qualquer fase: aguda ou crônica. A transmissão da doença da mãe para o filho, pode se dar em qualquer época da gestação, sendo mais provável no momento do parto, pelo contato das mucosas do feto com o sangue da mãe infectada.

Outras formas de transmissão são por via oral (na ingestão de alimentos contaminados), transmissão transfusional ou por transplante de órgãos.

A infecção causada pelo Trypanosoma Cruzi no ser humano ocorre em duas fases, e que são consideradas no modelo proposto: a fase aguda que pode ser tratada e a fase crônica.

A metodologia no estudo da modelagem matemática depende do problema a ser abordado. Neste trabalho estamos interessados na incidência da doença em indivíduos, e para isto fizemos uso de modelos

*bolsista UFABC 
de equações diferenciais ordinárias.

Deste modo, estudamos modelos propostos por alguns autores, discutimos alterações, até a elaboração e proposta de um modelo novo. Um dos modelos estudados para a dinâmica temporal, o qual foi proposto por Das e Mukherjee [3], considera a iteração entre o vetor e o homem. Utiliza também, para uma das taxas de contágio um termo que será reconsiderado em nosso trabalho, pois em nossas referências não encontramos indícios de que, uma pessoa que está na fase aguda da doença passa para a crônica através de um segundo contágio. O que acontece é que alguns indivíduos na fase aguda passam para a fase crônica e outros se recuperam por tratamento [2]. Consideramos isso para obter um modelo mais realista e assim obter estratégias de controle mais apropriadas. Então propomos o seguinte modelo:

$$
\begin{aligned}
& \frac{d S}{d t}=\mu H+q \gamma A+\theta C-p \frac{C}{H}-\frac{b \alpha}{H} S W-\mu S \\
& \frac{d A}{d t}=\frac{b \alpha}{H} S W-\gamma A+p \frac{C}{H}-\mu A \\
& \frac{d C}{d t}=(1-q) \gamma A-\theta C-\mu C \\
& \frac{d V}{d t}=\eta N-b\left(\frac{\beta_{1}}{H} A+\frac{\beta_{2}}{H} C\right) V-\eta V \\
& \frac{d W}{d t}=b\left(\frac{\beta_{1}}{H} A+\frac{\beta_{2}}{H} C\right) V-\eta W
\end{aligned}
$$

Onde as variáveis $H$ e $N$ denotam a população total de indivíduos hospedeiros e de vetores respectivamente, $S$ e $V$ as subpopulações de indivíduos suscetíveis e vetores suscetíveis da população total, $A$ e $C$ subpopulação de indivíduos na fase aguda e na fase crônica da doença respectivamente. Denotamos por $W$ a subpopulação de vetores infectados.

A taxa de mortalidade em vetores é denotada por $\eta$ e para humanos por $\mu$. Os parâmetros $\alpha, \beta_{1}$, $\beta_{2}$ são as probabilidades de transmissão da doença de vetor para humano, e de humano na fase aguda e crônica para vetor. A taxa de transmissão vertical é denotada por $p, b$ média diaria de picadas por vetor, $q \gamma$ taxa de indivíduos recuperados na fase aguda da doença, $(1-q) \gamma$ taxa de indivíduos que passam da fase aguda para a fase crônica da doença.

Determinamos o Número de Reproditibilidade Basal $R_{0}$, através do qual a dinâmica do sistema fica determinada. Através do estudo deste sistema de equações diferenciais avaliamos os resultados encontrados e obtemos possíveis estratégias de controle ao vetor.

Palavras-chave: Doença de Chagas, Epidemiologia, Modelagem Matemática

\section{Referências}

[1] A. M. Argolo; M. Felix; R. Pacheco; J. Costa, "Doença de Chagas e seus principais vetores no Brasil”, Fundação Oswaldo Cruz, Rio de Janeiro, 2008.

[2] G. Cruz-Pacheco; L. Esteva; C. Vargas, Control measures for Chagas' disease, Mathematical Biosciences, vol. 237, pp. 49-69, (2012).

[3] P. Das; D. Mukherjee, Qualitative study of a model of Chagas' disease, Mathematical and Computer Modelling, vol.43, pp. 413-422, (2006).

[4] J. D. Murray, "Mathematical Biology”, Springer, Berlin, 2002.

[5] J. X. Velasco-Hernandez, An epidemiological model for the dynamics of Chagas' disease, ByoSistems, vol.26, pp. 127-134, (1991). 